\title{
Evaluación de Collect Earth como herramienta para estimar datos de actividad para el programa REDD+ de Costa Rica
}

\author{
Evaluating Collect Earth as a tool to estimate activity data for Costa Rica's \\ REDD+ program
}

\author{
David Romero-Badilla ${ }^{1} \bullet$ Edgar Ortiz-Malavasi ${ }^{2} \bullet$ German Obando-Vargas ${ }^{1} \bullet$ \\ Héctor Arce-Benavides ${ }^{3} \bullet$ Maria Elena Herrera-Ugalde ${ }^{3}$ Guisella Quirós-Ramírez ${ }^{3}$
}

\begin{abstract}
This study examines the capacity of the software Collect Earth to generate activity data as required by Costa Rica's REDD+ program. Thereby, estimations of land use changes for the 2014-2015 period were made according to the Costa Rica's REDD+ monitoring, reporting and verification (MRV) system. Land use data was obtained through multitemporal visual interpretation of sampling points using high resolution satellite imagery via Collect Earth. Estimations of areas of change were made in accordance with general REDD+ land use change categories and REDD+ CR activity classes. Evaluation of two different types of stratifications were also made. The study demonstrates that it is possible to estimate activity data following the REDD+ CR MRV system using Collect Earth. However, it is necessary to intensify the sampling in areas of interest in order to reduce uncertainties, as well as to increase accessibility the high resolution imagery in Collect Earth to decrease the number of sample points without data.
\end{abstract}

Key words: Multitemporal visual interpretation, high resolution satellite imagery, land use changes, deforestation, reference data.

1. Consultor independiente; Cartago, Costa Rica;

darbafc@gmail.com, gobando67@gmail.com

2. Escuela de Ingeniería Forestal, Tecnológico de Costa Rica;

Cartago, Costa Rica; eortiz@itcr.ac.cr

Recibido: 13/03/2019

3. Secretaría REDD+, Fondo Nacional de Financiamiento

Aceptado: 13/06/2019

Forestal; San José, Costa Rica; harce@fonafifo.go.cr,

Publicado: 28/06/2019

mherrera@fonafifo.go.cr, gquiros@fonafifo.go.cr

DOI: $10.18845 /$ rfmk.v17i40.4904 
Con el fin de demostrar la capacidad de la herramienta Collect Earth para generar datos de actividad para el programa REDD+ Costa Rica, se realizaron estimaciones de uso y cambios de uso de la tierra en Costa Rica para el periodo 2014-15 según los enfoques de cálculo del sistema de medición, reporte y verificación (MRV) del programa REDD+ de Costa Rica. Los datos de uso de la tierra fueron obtenidos mediante evaluación visual multitemporal (EVM) de puntos de muestreo utilizando imágenes satelitales de alta resolución a través de Collect Earth. Las estimaciones de áreas de cambio de uso se realizaron según categorías generales de cambio, y según las actividades específicas de REDD+, además de evaluar distintos tipos de estratificaciones. Se demostró que es posible hacer las estimaciones de datos de actividad requeridas para el MRV de REDD+ CR a partir de Collect Earth, sin embargo, es necesario intensificar el muestreo en las áreas de interés para reducir las incertidumbres, y mejorar la disponibilidad de imágenes de alta resolución en Collect earth para disminuir la cantidad de puntos clasificados como "sin información".

Palabras clave: Evaluación visual multitemporal, imágenes satelitales de alta resolución, cambio de uso de la tierra, deforestación, datos de referencia.

\section{Introducción}

El mecanismo de reducción de emisiones por deforestación y degradación de los bosques, más la conservación y aumento de reservas de carbono forestal (REDD+) ha surgido como una estrategia clave para la mitigación del cambio climático [1]. Se trata de una iniciativa de la Convención Marco de las Naciones Unidas sobre el Cambio Climático (CMNUCC) que pretende reducir las emisiones de gases de efecto invernadero (GEI) concediendo un valor financiero al carbono almacenado en los bosques y brindando incentivos económicos para los países en desarrollo participantes [2], [3].

El monitoreo de emisiones y remociones GEl en los bosques, mediante metodologías robustas, es fundamental para el éxito de REDD+ [4]. Los países participantes como parte de su "preparación" para REDD+ deben desarrollar, entre otros productos, una cuantificación de las emisiones históricas nacionales, conocida como Nivel de Referencia de Emisiones Forestales (NREF) [3], y un Sistema Nacional de Monitoreo Forestal (SNMF) que integre un apropiado sistema de monitoreo y Medición, Reporte y Verificación (MRV) para proveer información periódica, transparente y consistente sobre los resultados obtenidos a través de las medidas y políticas nacionales implementadas para REDD+ [2], [3], [5]. En Costa Rica, la coordinación del proceso de preparación de REDD+ está a cargo del Fondo Nacional de Financiamiento Forestal (FONAFIFO), mientras que el SNMF está integrado por el Sistema Nacional de Monitoreo de Cobertura y Uso de la Tierra, y Ecosistemas (SIMOCUTE) [6].

Para el cálculo de emisiones de GEl se debe estimar y monitorear dos variables clave: (i) datos de actividad; y (ii) factores de emisión [4], [7]. Los datos de actividad se definen como la magnitud de la actividad humana generadora de emisiones $o$ remociones en un determinado periodo de tiempo [8], que en el caso de REDD+ se refiere al área de extensión de las actividades REDD+ expresado en hectáreas por año [3]. Los métodos de estimación de datos de actividad para REDD+ deben ser coherentes con la orientación de buenas prácticas del Panel Intergubernamental de expertos sobre el Cambio Climático (IPCC) para inventarios de gases de efecto invernadero, lo cual implica que se deben satisfacer dos criterios: (i) las estimaciones no son excesivas ni escasas, en la medida en que se pueda determinar, y (ii) las incertidumbres se deben minimizar tanto como sea posible [5], [9].

Históricamente, la generación de datos de actividad para REDD+ se ha basado en la elaboración de mapas de uso o cambio de uso de la tierra a partir de imágenes satelitales, a partir de los cuales se obtienen las áreas de cambio mediante el conteo de pixeles asignados a las distintas clases [4], [10], y es precisamente este método el que ha utilizado Costa Rica para hacer las estimaciones de su programa REDD+ [2]. Sin embargo, se ha manifestado que este método conlleva sesgo debido a que no considera errores de clasificación $y$, además, no genera directamente la información necesaria para construir intervalos de confianza, por lo que estos métodos no presentan la robustez estadística para garantizar que se cumpla con las directrices del IPCC [5], [11], [12].

Como alternativa, los países están implementando progresivamente los enfoques de muestreo de referencia para estimar datos de actividad [10], [13]. Uno de estos enfoques es la evaluación visual multitemporal (EVM) usando puntos de muestreo sobre imágenes de alta resolución. Es así como Collect Earth ha surgido como una herramienta de suma utilidad para el MRV de REDD+. Collect Earth es un software nuevo, de carácter gratuito y código libre, desarrollado por la Organización de las Naciones Unidas para la Agricultura y la Alimentación (FAO), que permite realizar muestreos 
por puntos para la recolección y análisis del uso de la tierra mediante la Evaluación Visual Multitemporal (EVM) de imágenes de alta y muy alta resolución, que cumplan con los requerimientos de monitoreo de REDD+ [14], permitiendo modificar el diseño de muestreo, el tamaño de las parcelas, y la forma de recolección de datos para ajustarse a las necesidades específicas de cada país [15].

Ante esto, y como parte de su enfoque progresivo, Costa Rica busca evaluar nuevos métodos de estimación de datos de actividad que sean consistentes con los objetivos de su programa REDD+ y con las orientaciones metodológicas del IPCC. Por tanto, este trabajo tiene como objetivo general demostrar la capacidad de Collect Earth para estimar datos de actividad en el programa REDD+ de Costa Rica, y como objetivo específico: (i) estimar datos de actividad según los sistemas de cálculo diseñados para el programa REDD+ CR a partir de datos de uso existentes del periodo 2014-2015.

\section{Materiales y métodos}

\section{Sitio de estudio}

Costa Rica es un país de América Central, ubicado entre las coordenadas geográficas $8^{\circ} 02^{\prime}$ y $11^{\circ} 13^{\prime}$ de latitud Norte y los $82^{\circ} 34^{\prime}$ y $85^{\circ} 58^{\prime}$ de longitud Oeste, limitando al Norte con Nicaragua, al Sureste con Panamá, al Oeste con el Océano Pacífico y al Este con el mar Caribe (figura 1) [16]. El área continental del país según el NREF emitido por Costa Rica ante la CMNUCC [17] corresponde a 5 133939,50 ha.

A pesar de ubicarse en la franja neotropical, el país posee una gran variedad de climas que dan lugar a 12 distintas zonas de vida según la clasificación de Holdridge [18]. El promedio anual de temperatura oscila entre $26^{\circ} \mathrm{C}$ en la costa caribe y $27,8^{\circ} \mathrm{C}$ en la costa pacífica, hasta $4,5^{\circ} \mathrm{C}$ en el punto más alto; mientras que la precipitación anual oscila entre 1400 y $8000 \mathrm{~mm}$ con un promedio a nivel nacional de $3300 \mathrm{~mm}$ anuales [19].

\section{Datos de uso de la tierra}

Se utilizaron los datos de uso de la tierra en Costa Rica recolectados por FONAFIFO para el periodo 2014-2015. Estos datos fueron generados mediante evaluación visual multitemporal de puntos de muestreo con imágenes satelitales por medio de la plataforma Collect Earth, y se siguiendo el protocolo de etiquetado para la interpretación visual de puntos de muestreo para uso de la tierra realizado por Tapia y Ortiz [20].

El muestreo se realizó a partir de la malla de puntos adoptada por el SIMOCUTE (figura 2) la cual contiene 10166 puntos con sistema de coordenadas sin proyectar

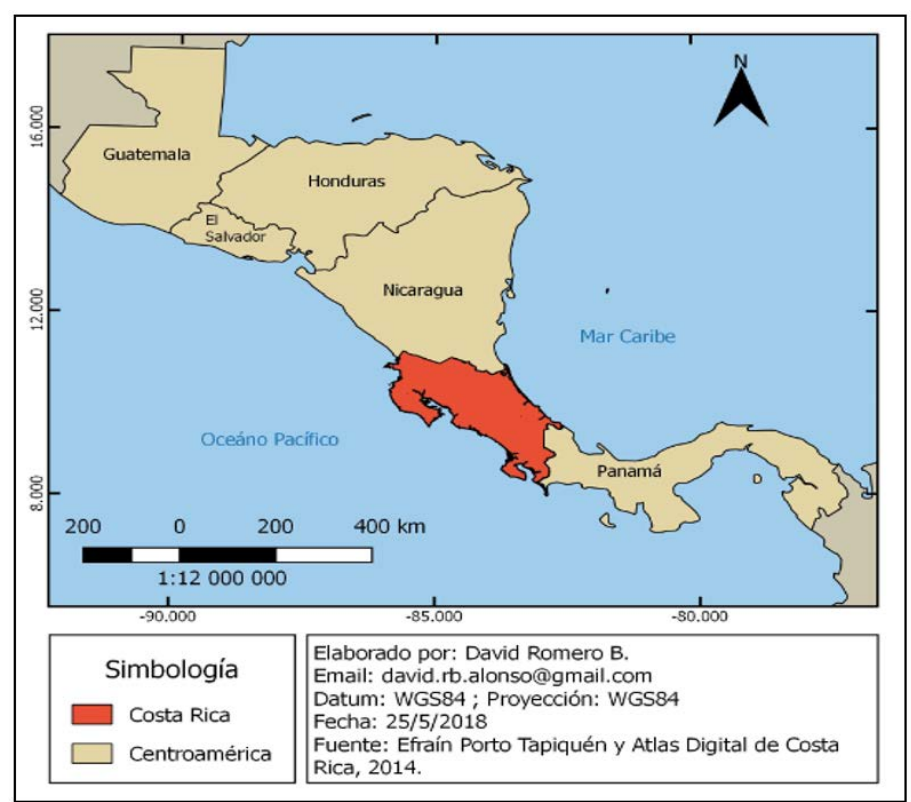

Figura 1. Ubicación de Costa Rica en Centroamérica.

Figure 1. Location of Costa Rica in Central America.

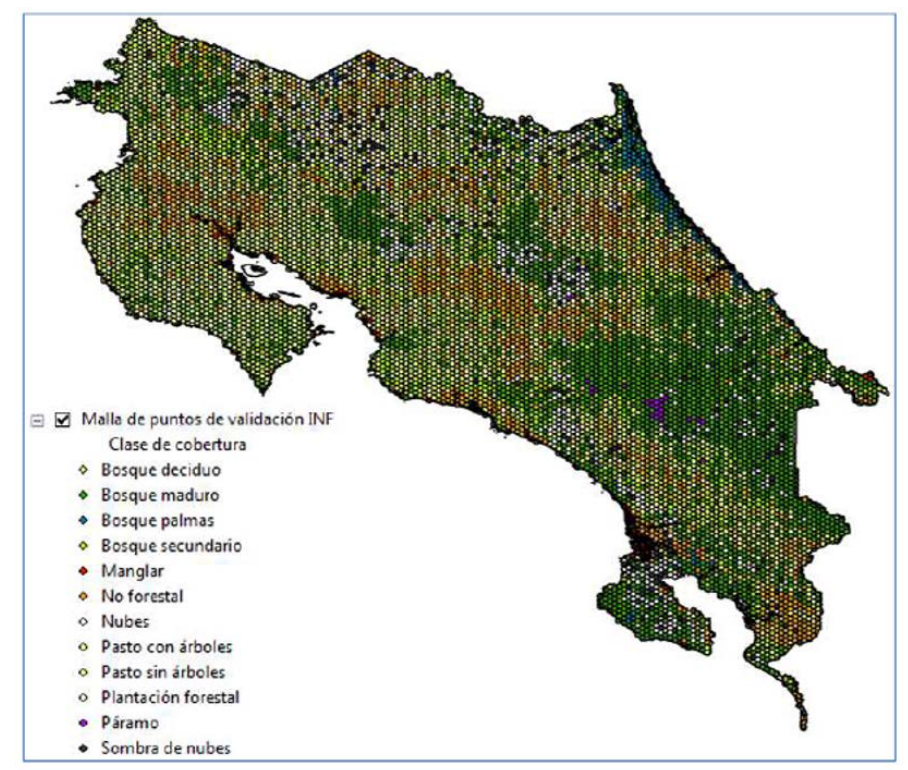

Figura 2. Malla de puntos adoptada por el Sistema Nacional de Monitoreo de la Cobertura y Uso de la Tierra y Ecosistemas en Costa Rica. Fuente: [22].

Figure 2. Grid of points adopted by Costa Rica's National Land Cover, Land Use, and Ecosystems Monitoring System. Source: [22].

WGS84, obtenidos a partir de la malla triangular regular con 2 402,5 metros de distancia entre puntos, propuesta por Ortiz [21] para el inventario forestal nacional de Costa Rica.

En cada punto de la malla se generó una parcela de muestreo de Collect Earth de 0,98 ha con forma cuadrada 
y con una grilla de $7 \times 7$ sub-puntos dentro de esta (ver figura 3). Así, el registro de uso de la tierra en cada parcela se realizó considerando todos los sub-puntos de la grilla, registrando entonces todos los usos dentro de la parcela a manera de porcentajes. En cada una de las parcelas, utilizando una plantilla de encuesta de Collect Earth, se registró la información sobre: (i) imágenes utilizadas, (ii) fecha de referencia, (iii) uso de la tierra dentro de la parcela, (iv) existencia y causa de cambio de uso de la tierra, (v) nivel de confianza de la interpretación, y (vi) observaciones importantes. Se utilizaron diferentes tipos imágenes para la interpretación de uso de la tierra, y su empleo dependió de la disponibilidad de estas. El orden de prioridad fue: (1) Imágenes satelitales de alta resolución de Digital Globe (Google Earth) o Bing Maps; (2) Imágenes satelitales de mediana resolución de Sentinel 2 y; (3) Imágenes satelitales de mediana resolución de Landsat.

La información recolectada, fue guardada como archivos CSV delimitado por comas que posteriormente fueron transformados a hojas de cálculo del programa Microsoft Excel para realizar el análisis.

\section{Estimación de datos de actividad REDD+ para Costa Rica}

Para la estimación de las áreas de cambio de uso de la tierra en Costa Rica durante el periodo 2014-2015, primeramente, se asignó una única categoría de uso de la tierra a cada parcela a partir de los diferentes porcentajes de uso registrados en cada una de ellas. Para esto, se consideró la definición de bosque utilizada en el NREF emitido por Costa Rica ante el FCPF [17], por lo que se utilizó el siguiente criterio: si la parcela presentaba al menos un $30 \%$ de uso de bosque, se asignó el tipo de bosque con mayor porcentaje; de lo contrario, se asignó el uso que presentara mayor porcentaje dentro de la parcela. Luego los usos asignados en ambos años a cada parcela fueron comparados mediante una tabulación cruzada para determinar los cambios de uso ocurridos en el periodo de estudio. Estos cambios fueron agrupados en primera instancia según las categorías generales de cambio de REDD+ las cuales se muestran en el cuadro 1, y se estimó las áreas de estas y sus respectivos errores y límites de confianza. Este análisis se realizó dividiendo los datos en 4 distintos grupos para examinar fuentes de variación en las estimaciones de áreas de cambio, a saber:

a) Escenario 1: conjunto total de puntos de muestreo.

b) Escenario 2: el grupo de datos con información de imágenes de alta resolución.

c) Escenario 3: el grupo de datos con información de imágenes de alta resolución y que presentan un desplazamiento entre imágenes no mayor a $7 \mathrm{~m}$.

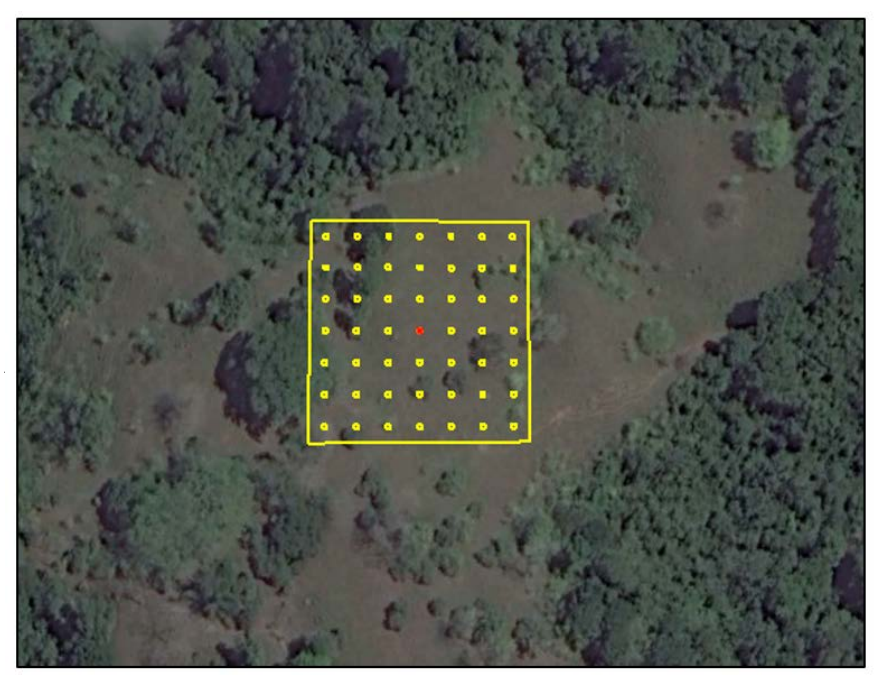

Figura 3. Ejemplo de la parcela de muestreo utilizada en este estudio.

Figure 3. Example of the sampling plot used in this study].

d) Escenario 4: el grupo de datos con información de imágenes de alta resolución, que presentan un desplazamiento entre imágenes no mayor a $7 \mathrm{~m}$ y cuyas fechas se encuentran en dentro del rango de enero 2012 a junio 2014 para el año 2014 y de julio 2014 a diciembre 2016 para el año 2015.

La decisión de establecer un límite de máximo $7 \mathrm{~m}$ de desplazamiento se debe a que esta es la distancia que existe entre cada punto de la grilla.

Posteriormente se utilizó el grupo de datos del escenario 4 , debido a que se consideró este como el escenario ideal, para hacer estimaciones de áreas, errores y límites de confianza de:

i. Cambios de uso según las categorías generales de cambio REDD+4 estratificado según áreas protegidas y no protegidas.

\section{ii. Actividades REDD+.}

\section{iii. Actividades REDD+ por tipo de bosque.}

Las actividades REDD+ consideradas en este trabajo se muestran en el cuadro 2.

La estratificación por tipos de bosque se realizó considerando los mismos tipos de bosque considerados en el NREF de Costa Rica [17], los cuales fueron: bosques muy húmedos y pluviales (Bhp), bosques húmedos (Bh), bosques secos (Bs), manglares (Ma) y bosques de palma (By). Para asignar cada parcela a un tipo de bosque se utilizó el mapa de tipos potenciales de bosque mostrado en la figura 4. Por su parte, la estratificación por área protegida consideró dos estratos: (i) área protegida, 
Cuadro 1. Descripción de las categorías generales de cambio de uso REDD+.

Table 1. Description of the REDD+ general land use change categories.

\begin{tabular}{|ccr|}
\hline Categoría de cambio & Acrónimo & Descripción \\
\hline Bosque estable & F-F & Tierras forestales que se mantienen como tales \\
\hline Deforestación & F-N & Tierras forestales convertidas a tierras no forestales \\
\hline No bosque estable & N-N & Tierras no forestales que se mantienen como tales \\
\hline Nuevos bosques & N-F & Tierras no forestales convertidas a tierras forestales \\
\hline Sin información & ND & Aquellas parcelas en las que en algún año se le asignó el uso de \\
& & nubes, sombras o sin información
\end{tabular}

Cuadro 2. Actividades REDD+ consideradas en el estudio.

Table 2. REDD+ activities considered in this study.

Simbología

\begin{tabular}{|c|c|}
\hline \multicolumn{2}{|l|}{ DF } \\
\hline $\mathrm{NL}$ & Pérdida \\
\hline $\mathrm{CO}$ & Conservación \\
\hline AE.ff & Aumento de existencias de carbor \\
\hline AE.nf & Aumento de existencias de \\
\hline AE.pp & Aumento de existencias de carbono foresta \\
\hline AE.np & Aumento de existencias de carbon \\
\hline EXL & Áreas sin infor \\
\hline NA & Áreas sin existencias de \\
\hline $\begin{array}{l}\text { en el cual } \\
\text { protegidas } \\
\text { categoría d } \\
\text { todas las } \\
\text { protegidas. } \\
\text { la capa de } \\
\text { Costa Rica }\end{array}$ & $\begin{array}{l}\text { nsideraron todas las áreas silvestres } \\
\text { ntes en Costa Rica sin importar su } \\
\text { ejo y; (ii) área no protegida, que fueron } \\
\text { s ubicadas fuera de áreas silvestres } \\
\text { Stratificar por área protegida se utilizó } \\
\text { Silvestre Protegidas de Atlas Digital de }\end{array}$ \\
\hline
\end{tabular}

Las fórmulas utilizadas para estimar las áreas de cambio, sus errores y límites de confianza asociados fueron la ecuación 1 a la 6.

$$
\begin{gathered}
p_{i}=\frac{n_{i}}{N} \\
A_{i}=A * p_{i} \\
S_{A i}=\sqrt[2]{\frac{p_{i^{*}\left(1-p_{i}\right)}}{N}} * A \\
E(\%)_{A i}=\frac{S_{A i * 1,96}}{A_{i}} * 100 \\
L i_{i}=A_{i}-E_{A i} \\
L s_{i}=A_{i}+E_{A i}
\end{gathered}
$$

Descripción

Deforestación

érdidas no antrópicas de bosques

de existencias de carbono forestal.

no forestal en bosques que permanecen como bosques

arbono forestal en tierras convertidas a bosques forestales

Áreas sin información excluidas de la contabilidad

bono forestal donde REDD+ no es aplicable

Donde:

pi $=$ proporción de puntos de la clase de cambio i.

$\mathrm{ni}=$ cantidad de unidades de muestreo pertenecientes a la clase de cambio i.

$\mathrm{N}$ = cantidad total de unidades de muestreo.

$\mathrm{A} \mathrm{i}=$ área de la clase de cambio $\mathrm{i}$ en ha.

$A=$ área total de estudio $=5113939,5$ ha.

$\mathrm{SAi}=$ error estándar de la estimación del área de la clase de cambio $\mathrm{i}$.

$\mathrm{EAi}=$ error absoluto de la estimación del área de la clase de cambio i con una confiabilidad de $95 \%$.

$\mathrm{E}(\%) \mathrm{Ai}=$ error relativo de la estimación del área de la clase de cambio i con una confiabilidad de $95 \%$.

Lii = Límite inferior de confianza al $95 \%$ para la clase de cambio i.

Lsi = Límite superior de confianza al $95 \%$ para la clase de cambio i. 
Cuadro 3. Estimación del área y límites de confianza para los cambios de uso de REDD+ en Costa Rica en el periodo 2014-2015, bajo distintos escenarios.

Table 3. Area estimation and confidence intervals for REDD+ land use changes in Costa Rica during the 2014-2015 period, under different scenarios.

\begin{tabular}{|c|c|c|c|c|c|c|c|}
\hline Escenario6 & Cambio & $n_{i}$ & $p_{i}$ & $A_{i}$ & $E(\%)$ & $\mathrm{Li}$ & Ls \\
\hline \multirow{5}{*}{ Escenario 1} & F-N & 41 & 0,4 & 20624,8 & 30,5 & 14324,3 & 26925,3 \\
\hline & F-F & 3656 & 36,0 & 1839126,8 & 2,6 & 1791420,0 & 1886833,6 \\
\hline & $\mathrm{N}-\mathrm{F}$ & 84 & 0,8 & 42255,6 & 21,3 & 33256,5 & 51254,8 \\
\hline & $\mathrm{N}-\mathrm{N}$ & 2572 & 25,3 & 1293827,7 & 3,3 & 1250610,4 & 1337045,0 \\
\hline & ND & 3813 & 37,5 & 1918104,6 & 2,5 & 1869975,3 & 1966233,9 \\
\hline \multirow{5}{*}{ Escenario 2} & F-N & 37 & 0,7 & 33324,4 & 32,1 & 22621,6 & 44027,2 \\
\hline & $F-F$ & 3310 & 58,3 & 2981 179,9 & 2,2 & 2915592,1 & 3046767,8 \\
\hline & $\mathrm{N}-\mathrm{F}$ & 32 & 0,6 & 28821,1 & 34,6 & 18863,3 & 38778,9 \\
\hline & $\mathrm{N}-\mathrm{N}$ & 2284 & 40,2 & 2057104,2 & 3,2 & 1991878,0 & 2122330,5 \\
\hline & ND & 15 & 0,3 & 13509,9 & 50,5 & 6682,0 & 20337,8 \\
\hline \multirow{5}{*}{ Escenario 3} & F-N & 25 & 0,5 & 25437,4 & 39,1 & 15490,8 & 35384,1 \\
\hline & $F-F$ & 3006 & 59,8 & 3058595,7 & 2,3 & 2989277,4 & 3127914,1 \\
\hline & $\mathrm{N}-\mathrm{F}$ & 21 & 0,4 & 21367,4 & 42,7 & 12247,5 & 30487,3 \\
\hline & $\mathrm{N}-\mathrm{N}$ & 1959 & 39,0 & 1993276,5 & 3,5 & 1924323,6 & 2062 229,3 \\
\hline & ND & 15 & 0,3 & 15262,5 & 50,5 & 7550,1 & 22974,8 \\
\hline \multirow{5}{*}{ Escenario 4} & F-N & 13 & 0,3 & 17209,7 & 54,3 & 7870,2 & 26549,3 \\
\hline & $F-F$ & 2270 & 58,8 & 3005084,8 & 2,6 & 2925698,5 & 3084471,1 \\
\hline & $\mathrm{N}-\mathrm{F}$ & 11 & 0,3 & 14562,1 & 59,0 & 5968,7 & 23155,5 \\
\hline & $\mathrm{N}-\mathrm{N}$ & 1558 & 40,3 & 2062520,8 & 3,8 & 1983408,5 & 2141633,0 \\
\hline & ND & 11 & 0,3 & 14562,1 & 59,0 & 5968,7 & 23155,5 \\
\hline
\end{tabular}

Cuadro 4. Áreas y límites de confianza para las actividades REDD+ en Costa Rica en el periodo 2014-2015.

Table 4. Areas and confidence intervals of the REDD+ activities in Costa Rica during the 2014-2015 period.

\begin{tabular}{|cccccc|}
\hline Actividad REDD+ & $n_{i}$ & $A_{i}$ & $E(\%)$ & $L i$ & LS \\
\hline DF & 11 & 14562 & 59 & 5969 & 23156 \\
\hline NL & 2 & 2648 & 139 & -1021 & 6316 \\
\hline AE.ff & 1103 & 1460180 & 5 & 1387340 & 1533019 \\
\hline AE.nf & 8 & 10591 & 69 & 3259 & 17922 \\
\hline AE.pp & 139 & 184012 & 16 & 153976 & 214047 \\
\hline AE.np & 3 & 3971 & 113 & -521 & 8464 \\
\hline CO & 1028 & 1360893 & 5 & 1289624 & 1432161 \\
\hline EXL & 11 & 2562 & 59 & 5969 & 23156 \\
\hline NA & 1558 & 2062521 & 4 & 1983408 & 2141633 \\
\hline
\end{tabular}


Cuadro 5. Conservación de reservas de carbono en bosques maduros.

Table 5. Conservation of forest carbon stocks in primary forests.

\begin{tabular}{ccccccc|}
$\begin{array}{c}\text { Actividad } \\
\text { REDD+ }\end{array}$ & Tipo de bosque & ni & Ai (ha) & E(\%) & Li & Ls \\
\hline & Bhp & 531 & 1080529 & 6 & 1015882 & 1145176 \\
& Bh & 378 & 388580 & 9 & 352460 & 424700 \\
CO & Bs & 22 & 23658 & 38 & 14684 & 32632 \\
& Ma & 38 & 39384 & 17 & 32887 & 45882 \\
& By & 58 & 100079 & 17 & 82747 & 117411 \\
& Total & 1027 & 1632231 & 8 & 1498660 & 1765801 \\
\hline
\end{tabular}

Cuadro 6. Pérdidas de carbono en Bosques primarios.

Table 6. Carbon stocks loss in primary forests.

\begin{tabular}{|c|c|c|c|c|c|c|}
\hline $\begin{array}{l}\text { Actividad } \\
\text { REDD+ }\end{array}$ & Tipo de bosque & ni & $\mathrm{Ai}(\mathrm{ha})$ & $E(\%)$ & $\mathrm{Li}$ & Ls \\
\hline \multirow{6}{*}{ DF } & Bhp & 0 & & & & \\
\hline & $\mathrm{Bh}$ & 1 & 1028 & 196 & -987 & 3042 \\
\hline & Bs & 0 & & & & \\
\hline & $\mathrm{Ma}$ & 0 & & & & \\
\hline & By & 0 & & & & \\
\hline & Total & 1 & 1028 & 196 & -987 & 3042 \\
\hline \multirow{6}{*}{ NL } & Bhp & 0 & & & & \\
\hline & $\mathrm{Bh}$ & 0 & & & & \\
\hline & Bs & 0 & & & & \\
\hline & $\mathrm{Ma}$ & 0 & & & & \\
\hline & By & 0 & & & & \\
\hline & Total & 0 & & & & \\
\hline Total & 1 & 1028 & 196 & -987 & 3042 & \\
\hline
\end{tabular}

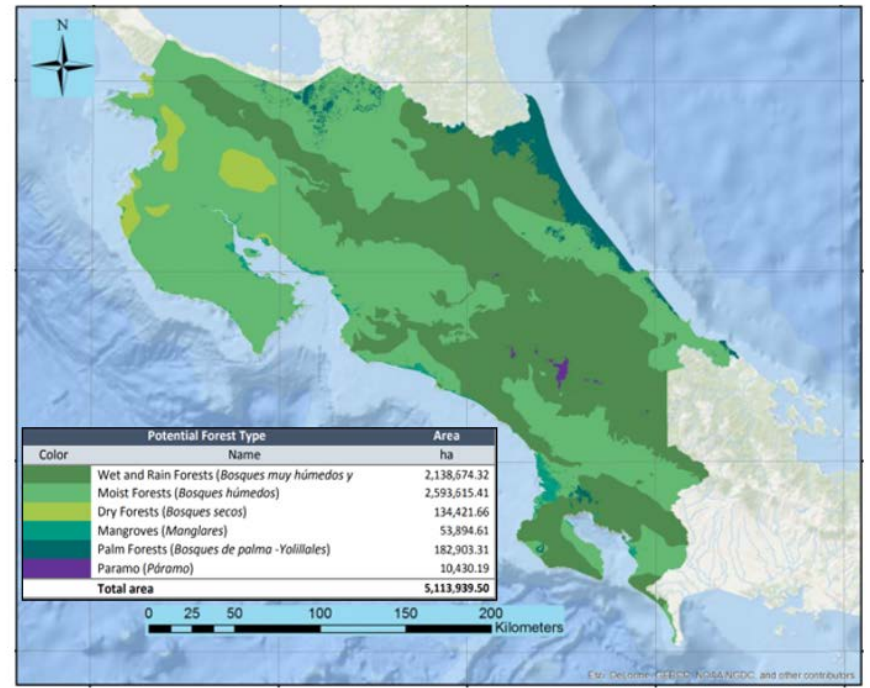

Figura 4.Mapa de tipos de bosque potenciales en Costa Rica. Fuente: [17].

Figure 4. Map of potential forest types in Costa Rica. Source: [17].

\section{Resultados y discusión}

\section{Estimación de datos de actividad REDD+ para Costa Rica}

En la estimación según las categorías generales de cambio de REDD+ las áreas variaron considerablemente según los distintos escenarios evaluados, tal como se puede observar en el cuadro 3 .

En el cuadro 3 se puede apreciar que, al utilizar únicamente los datos con información de imágenes de alta resolución (escenario 2), las áreas de las clases de bosque estable, no bosque estable y deforestación, aumentan considerablemente con respecto al escenario 1. Esto se debe principalmente a que la clase "sin información", que eran las que se presentaban en mayor cantidad en el escenario 1, disminuyeron de 3813 a tan solo 15 observaciones. 
Table 7. Carbon stocks loss in secondary forests.

\begin{tabular}{|c|c|c|c|c|c|c|}
\hline $\begin{array}{l}\text { Actividad } \\
\text { REDD+ }\end{array}$ & Tipo de bosque & ni & Ai (ha) & $E(\%)$ & $\mathrm{Li}$ & Ls \\
\hline \multirow{5}{*}{ DF } & Bhp & 4 & 8140 & 98 & 178 & 16101 \\
\hline & $\mathrm{Bh}$ & 5 & 5140 & 88 & 639 & 9641 \\
\hline & Bs & 0 & & & & \\
\hline & $\mathrm{Ma}$ & 0 & & & & \\
\hline & By & 0 & & & & \\
\hline \multirow{7}{*}{$\mathrm{NL}$} & Total & 9 & 13280 & 94 & 817 & 25742 \\
\hline & Bhp & 2 & 4070 & 138 & -1565 & 9705 \\
\hline & $\mathrm{Bh}$ & 0 & & & & \\
\hline & Bs & 0 & & & & \\
\hline & $\mathrm{Ma}$ & 0 & & & & \\
\hline & By & 0 & & & & \\
\hline & Total & 2 & 4070 & 138 & -1565 & 9705 \\
\hline Total & 11 & 17349 & 104 & -748 & 35447 & \\
\hline
\end{tabular}

Bajo el escenario 3, las áreas de deforestación y de bosques nuevos disminuyeron en 7887 ha y 7454 ha respectivamente, con respecto al escenario 2. Esto sugiere que el desplazamiento entre imágenes es un factor de error/sesgo importante en la estimación de cambios de uso. Probablemente el cambio registrado en parcelas con desplazamiento sea resultado de que no se está evaluando exactamente el mismo sitio.

Por su parte, bajo el escenario 4 las áreas de bosque estable y no bosque estable aumentan, tal como se esperaba, debido a que se acorta el rango de fechas en la evaluación dando paso a una menor cantidad de cambios de uso. El área de deforestación obtenida bajo el escenario 4, considerado el ideal, representa una disminución en poco más de la mitad con respecto a lo reportado en el NREF de Costa Rica [17]. EI NREF indica que hubo 34705 ha de deforestación total para el periodo 2012-2013 en comparación con las 17210 ha obtenidas en este estudio para el periodo 2014-2015, sin embargo, es probable que gran parte de esta diferencia sea atribuible a la diferencia en las metodologías de estimación.

Otro factor por tomar en cuenta con respecto a la variación según los distintos escenarios evaluados es que la cantidad de datos se va reduciendo significativamente, y la distribución de los datos que se excluyen no resulta completamente aleatoria. Esto puede conllevar a un sesgo asociado en las estimaciones que podría explicar las variaciones observadas.

Bajo todos los escenarios, el error en las clases de cambio estables (bosque estable y no bosque estable) fue menor a $4 \%$, mientras que en las clases de deforestación y nuevos bosques dadas las pocas observaciones que se logran obtener el error resulta sumamente alto, llegando a ser mayor al $50 \%$ en el escenario 4.

Al estratificar las categorías generales de cambio por áreas protegidas se obtuvo que, dentro de las áreas protegidas no hubo deforestación ni obtención de bosques nuevos, sino que estos cambios de uso se presentaron únicamente fuera de ellas. De acuerdo con estos resultados existen aproximadamente 1133 071 ha de bosque estable dentro de áreas protegidas en Costa Rica. Este dato, resulta inferior a lo indicado en el INF 2014-15 [22] donde se reporta un área de 1 265692 ha. Sin embargo, la estimación del INF abarca el área comprendida por la categoría de pastos con árboles. Esta estratificación por áreas protegidas resulta importante debido a que se ha determinado que en Costa Rica los bosques en áreas protegidas almacenan cerca de la mitad del $\mathrm{CO}_{2}$ capturado por especies vegetales mayores, y una tercera parte del $\mathrm{CO}_{2}$ fijado en el suelo en bosques [22].

En el cuadro 4, se observa que la mayoría de las deforestaciones ocurridas corresponden a deforestaciones de tipo antropogénico. Sin embargo, los errores son sumamente altos en las clases que implican un cambio de uso de la tierra (DF, NL, AE.nf, AE.np) de las cuales se obtuvieron pocas observaciones. Las demás actividades, por el contrario, presentaron errores muy bajos. 
Cuadro 8. Pérdidas de carbono en plantaciones forestales.

Table 8. Carbon stocks loss in forest plantations.

\begin{tabular}{|c|c|c|c|c|c|c|}
\hline $\begin{array}{c}\text { Actividad } \\
\text { REDD+ }\end{array}$ & Tipo de bosque & ni & $\mathrm{Ai}$ (ha) & $E(\%)$ & $\mathrm{Li}$ & Ls \\
\hline \multirow{5}{*}{ DF } & Bhp & 1 & 2035 & 196 & -1952 & 6021 \\
\hline & $\mathrm{Bh}$ & 0 & & & & \\
\hline & Bs & 0 & & & & \\
\hline & $\mathrm{Ma}$ & 0 & & & & \\
\hline & By & 0 & & & & \\
\hline \multirow{7}{*}{$\mathrm{NL}$} & Total & 1 & 2035 & 196 & -1952 & 6021 \\
\hline & Bhp & 0 & & & & \\
\hline & $\mathrm{Bh}$ & 0 & & & & \\
\hline & Bs & 0 & & & & \\
\hline & $\mathrm{Ma}$ & 0 & & & & \\
\hline & By & 0 & & & & \\
\hline & Total & 0 & & & & \\
\hline Total & & 1 & 2035 & 196 & -1952 & 6021 \\
\hline
\end{tabular}

Cuadro 9. Ganancias de carbono en bosques secundarios

Table 9. Enhancement of forest carbon stocks on secondary forest.

\begin{tabular}{|c|c|c|c|c|c|c|}
\hline $\begin{array}{l}\text { Actividad } \\
\text { REDD+ }\end{array}$ & Tipo de bosque & ni & $\mathrm{Ai}(\mathrm{ha})$ & $E(\%)$ & Li & Ls \\
\hline \multirow{5}{*}{ DF } & Bhp & 244 & 496514 & 11 & 441922 & 551106 \\
\hline & $\mathrm{Bh}$ & 781 & 802859 & 6 & 756071 & 849647 \\
\hline & Bs & 40 & 43015 & 26 & 32022 & 54008 \\
\hline & $\mathrm{Ma}$ & 4 & 4146 & 94 & 242 & 8049 \\
\hline & By & 33 & 56942 & 28 & 40819 & 73064 \\
\hline \multirow{7}{*}{ NL } & Total & 1102 & 1403476 & 9 & 1271077 & 1535874 \\
\hline & Bhp & 3 & 6105 & 113 & -794 & 13003 \\
\hline & $\mathrm{Bh}$ & 5 & 5140 & 87 & 639 & 9641 \\
\hline & Bs & 0 & & & & \\
\hline & $\mathrm{Ma}$ & 0 & & & & \\
\hline & By & 0 & & & & \\
\hline & Total & 8 & 11245 & 101 & -154 & 22643 \\
\hline Total & & 1110 & 1414720 & 10 & 1270922 & 1558518 \\
\hline
\end{tabular}

En el cuadro 5, se aprecia que se obtuvo un área de conservación de reservas de carbono en bosques maduros de 575360 ha, y esta proviene principalmente de los bosques húmedos. Para pérdidas de bosque primario solo se obtuvo una observación que resultó en un área de 1028 ha con un muy alto error (196 \%) (cuadro 6). Para bosques secundarios por su parte, se obtuvo una pérdida total de reservas de carbono de 17349 ha provenientes en su totalidad de bosques muy húmedos y pluviales y bosques húmedos, y en su mayoría tratándose de pérdidas de carácter antropogénico (cuadro 7), mientras que en plantaciones forestales se obtuvo tan solo una observación de pérdidas de reservas de carbono resultando en un área de 2035 ha y un error de $196 \%$ (cuadro 8). En cuanto a las ganancias en bosques secundarios se obtuvo un área de 1414720 ha donde el 99 \% de esta área proviene de bosques que permanecen como bosques (cuadro 9), mientras 
Table 10. Enhancement of forest carbon stocks on forest plantations.

\begin{tabular}{|c|c|c|c|c|c|c|}
\hline $\begin{array}{c}\text { Actividad } \\
\text { REDD+ }\end{array}$ & Tipo de bosque & ni & $\mathrm{Ai}(\mathrm{ha})$ & $E(\%)$ & Li & Ls \\
\hline \multirow{5}{*}{ DF } & Bhp & 10 & 20349 & 62 & 7797 & 32901 \\
\hline & $\mathrm{Bh}$ & 124 & 127471 & 17 & 105592 & 149349 \\
\hline & Bs & 5 & 5377 & 86 & 759 & 9995 \\
\hline & $\mathrm{Ma}$ & 0 & & & & \\
\hline & By & 0 & & & & \\
\hline \multirow{7}{*}{$\mathrm{NL}$} & Total & 139 & 153196 & 25 & 114148 & 192245 \\
\hline & Bhp & 1 & 2035 & 196 & -1952 & 6021 \\
\hline & $\mathrm{Bh}$ & 1 & 1028 & 196 & -987 & 3042 \\
\hline & Bs & 0 & & & & \\
\hline & $\mathrm{Ma}$ & 0 & & & & \\
\hline & By & 1 & 1725 & 195 & -1640 & 5091 \\
\hline & Total & 3 & 4788 & 196 & -4579 & 14155 \\
\hline Total & & 142 & 157985 & 31 & 109570 & 206400 \\
\hline
\end{tabular}

que en el área de ganancia de reservas de carbono en plantaciones forestales fue de 157985 ha donde el $97 \%$ de esta área proviene de plantaciones forestales estables (cuadro 10).

El presente trabajo evidencia que es posible realizar estimaciones de cambio de uso desde un nivel general hasta estimaciones específicas de diferentes actividades REDD+ a partir de datos tomados con la herramienta Collect Earth. Además, también fue posible aplicar diferentes estratificaciones a partir de capas de información geográfica. De esta manera se demuestra, la capacidad del Collect Earth para la estimación de datos de actividad para el MRV del programa REDD+ de Costa Rica.

Sin embargo, en todas las estimaciones de áreas de pérdidas y ganancias de bosques, los errores asociados son sumamente elevados (mayores al 50 \%). El FCPF sugiere que las incertidumbres se mantengan por debajo del $15 \%$ [24], por lo que se deben buscar formas de reducir el error de las estimaciones. Partiendo de los principios generales del muestreo, se sabe que un número mayor de parcelas produce errores más bajos de muestreo [25], y como se puede apreciar en los resultados, fueron muy escasas las observaciones que se obtuvieron para las clases de cambio que involucran tierras forestales convertidas a tierras no forestales, y tierras no forestales convertidas a tierras forestales. Este problema se amplía al estratificar por tipo de bosque y al hacer estimaciones más específicas por actividad REDD+. Casanoves et al. [26], sugiere que se debe buscar un punto de equilibrio entre la precisión y el costo que implica la toma de datos, al mismo tiempo que se satisfagan los requerimientos de los usuarios finales.

Hewson et al. [25], indica que es común realizar estratificaciones por tipo de bosque, dado que este enfoque aumenta el poder estadístico y brinda estimaciones más precisas de las reservas de carbono. Sin embargo, el autor indica que, si el objetivo del muestreo es cuantificar con precisión los cambios en las reservas de carbono forestal, los esfuerzos se deben enfocar a asignar parcelas en áreas que las reservas de carbono son susceptibles a disminuir (degradación o deforestación) o aumentar (reforestación o regeneración). Respecto a esto, el GFOI [5] menciona que se pueden utilizar datos auxiliares para detectar o pronosticar áreas de cambio en las cuales se puede implementar la intensificación del muestreo para mejorar las estimaciones de interés particular. Estos datos pueden ser: historiales de alteraciones, tenencia de la tierra, estadísticas de explotación de bosques, datos sobre incendios forestales, datos sobre el impacto de plagas, entre otros, que se tengan en formato espacial. Además, el GFOI [5] agrega que este puede ser un método eficiente en relación con los costos. En el presente estudio, por ejemplo, los resultados obtenidos al estratificar por área protegida sugieren que la información de áreas protegidas puede ser información de utilidad dado que la deforestación y ganancia de nuevos bosques ocurrieron fuera de ellas. Otro factor por considerar es que la gran cantidad de datos sin información redujo en una tercera parte el tamaño de la muestra, lo cual reduce la robustez 
del muestreo significativamente y además es posible que genere un sesgo asociado a una distribución no aleatoria de los datos restantes. Esto demuestra que es necesario mejorar la disponibilidad de imágenes de alta resolución en la plataforma de Collect Earth, ya sea adquiriendo fotografías adicionales para agregar en la herramienta externamente, o mediante convenios con Google Earth.

\section{Conclusiones}

Es posible utilizar datos de EVM obtenidos a través de Collect Earth para realizar estimaciones de uso y cambios de uso de la tierra a diferentes niveles de especificidad y aplicando diferentes estratificaciones para ajustar las estimaciones según los requerimientos del programa REDD+ Costa Rica.

La resolución de las imágenes utilizadas, el desplazamiento entre imágenes y el rango de fechas utilizadas tienen un efecto considerable en las estimaciones.

Los errores de estimación para las categorías de bosques nuevos y deforestación fueron mayores al $50 \%$, y al hacer estimaciones por actividad REDD+ y estratificar por tipo de bosque estos incrementan aún más. Esto debido a que la cantidad de observaciones para estas actividades tan específicas resultan muy escasas, y para corregir esto es necesario establecer un diseño de muestreo en función de la detección de estas áreas de interés.

\section{Recomendaciones}

Incorporar el uso de datos auxiliares de fotografías de alta resolución, como historiales de alteraciones, tenencia de la tierra, estadísticas de explotación de bosques, entre otros, que se tengan en formato espacial. Esto con el fin de determinar áreas de posible pérdida o ganancia de reservas forestales de carbono, de manera que se pueda ajustar el diseño de muestreo, intensificando la muestra en estas áreas, con el fin de obtener una mayor cantidad de observaciones en las áreas de interés y obtener estimaciones más precisas.

\section{Referencias}

[1] Secretaría REDD+ Costa Rica, "Plan de implementación de la Estrategia Nacional REDD+ Costa Rica", San José, Costa Rica, 2017. Disponible en http://reddcr.go.cr/sites/default/ files/centro-de-documentacion/plan_de_implementacion_ enreddcr_v3.pdf

[2] M. J. Mlotha, "Analysis of Land Use/Land Cover Change Impacts Upon Ecosystem Services in Montane Tropical
Forest of Rwanda: Forest Carbon Assessment and REDD+ Preparedness", Tesis, Antioch University, New England, 2018.

[3] M. Köhl, C. Scott, A. Lister, I. Demon, D. Plugge, "Avoiding treatment bias of REDD+ monitoring by sampling with partial replacement", Carbon balance and management, vol. 10, No. 1, p. 11. DOI 10.1186/s13021-015-0020-y.

[4] V. De Sy et al., "Synergies of multiple remote sensing data sources for REDD+ monitoring", Current Opinion in Environmental Sustainability, vol. 4, núm. 6, pp. 696-706, 2012.

[5] GFOI (Global Forest Observations Initiative), "Integración de las observaciones por teledetección y terrestres para estimar las emisiones y absorciones de gases de efecto invernadero en los bosques: Métodos y Orientación de la Iniciativa Mundial de Observación de los Bosques" Edición 2.0, Organización de las Naciones Unidas para la Alimentación y la Agricultura, Roma. 2016

[6] Ministerio de Ambiente y Energía, "Propuesta para el diseño del Sistema Nacional de Monitoreo de Cobertura y Uso de la Tierra y Ecosistemas" Version 2. San José, 2017. Disponible en https://simocute.org/wp-content/uploads/2019/02/ Propuesta-SIMOCUTE-v4-1.pdf

[7] J. Fernandez, "Medición, reporte y verificación de REDD+: documentp metodológico". Secretaria REDD+ Costa Rica, 2013. Disponible en http://reddcr.go.cr/sites/default/files/ centro-de-documentacion/mrv.pdf

[8] IPCC (Intergovernmental Panel on Climate Change), "GoodPractice Guidance for Land Use, Land-Use Change and Forestry", Institute for Global Environmental Strategies, 2003.

[9] IPCC (Intergovernmental Panel on Climate Change), "Guidelines for National Greenhouse Gas Inventories", Vol 4, Agriculture, Forestry and other Land Use. Institute for Global Environmental, 2006.

[10] R. McRoberts, S.V. Stehman, G.C. Liknes, E. Naesset, C. Sannier \& B.F. Walters, "The effects of imperfect reference data on remote sensing-assisted estimators of land cover class proportions", ISPRS Journal of Photogrammetry and Remote Sensing, vol. 142, Agosto, 2018.

[11] R. E. McRoberts, "Satellite image-based maps: Scientific inference or pretty pictures?", Remote Sensing of Environment, vol. 115, no. 2, pp. 715-724, 2011.

[12] P. Olofsson, G.M. Foody, S. V. Stehman \& C.E Woodcock, "Making better use of accuracy data in land change studies: Estimating accuracy and area and quantifying uncertainty using stratified estimation", Remote Sensing of Environment, vol. 129, 15 feb., 2013.

[13] GFOI (Global Forest Observations Initiative). Summary of Country experiences and critical issues related to estimation of activity data, 2018. Disponible en https:// www.reddcompass.org/documents/184/0/ActivityData Inference_FAQ.pdf/8e93e100-c46b-4ff9-946b6d0972fd50da

[14] FAO (Organización de las Naciones Unidas para la Alimentación y la Agricultura), "Collect Earth User Manual: A guide to monitoring land use change and deforestation with free and open source software" 
[15] A. Bey, et al., "Collect Earth: Land Use and Land Cover Assessment through Augmented Visual Interpretation. Remote Sensing" 8(10): p 807-830 . 2016

[16] UNESCO (Organización de las Naciones Unidas para la Educación, la Ciencia y la Cultura). Balance hídrico superficial de Costa Rica. Período: 1970-2002. Documentos Técnicos del PHI-LAC, No1. Recuperado de: http://unesdoc.unesco. org/images/0015/001563/156338s.pdf. 2017

[17] MINAE (Ministerio de Ambiente y Energía). "Forest reference emission level/forest reference level Costa Rica: Submission to the UNFCCC secretariat for technical review according to decision 13/CP.19." 2016.

[18] Quesada, R, "Los Bosques de Costa Rica". Centro de investigación integración. 2007. Disponible en: http:// www.cientec.or.cr/exploraciones/ponencias2007/ RupertoQuesada.pdf

[19] FAO. "FRA 2000: Bibliografía comentada en Cambios en la cobertura forestal en Costa Rica." Roma: FAO. 2000.

[20] A. Tapia, E. Ortiz, "Protocolo para el etiquetado de referencia: cambios de uso de la tierra", 2017.

[21] E. Ortiz, "Cartografía base para realizar el Inventario Nacional Forestal (INF) de Costa Rica 2013-2014". REDD/CCAD/ GIZ. SINAC-FONAFIFO. San José, Costa Rica, 2014.

[22] MINAE (Ministerio de Ambiente y Energía) y SINAC (Sistema Nacional de Áreas de Conservación) Marco Metodológico para la Fase I (Pre-Muestro) y la Fase II (Muestreo). Inventario Forestal Nacional. Volumen 4/SINAC, Programa REDD/CCDA-GIZ, FONAFIFO. Compilado por Ing. Jorge Fallas Gamboa. San José, Costa Rica: Programa REDD/ CCDA-GIZ. 2015.

[23] E. Ortiz, "Atlas digital de Costa Rica", Escuela de Ingeniería Forestal, ITCR, 2014

[24] FCPF, "Marco metodológico del Fondo de Carbono del FCPF" Washington, D.C., The World Bank. 39 p, 2013.

[25] Hewson, J., M.K. Steininger y S. Pesmajoglou, A. Parra y D. Shoch. 2014. Manual de Medición, Reporte y Verificación (MRV) de REDD+ Versión 2.0: Resumen.

[26] F. Casanoves, M. Cifuentes, M. Chachón. "Estimación del carbono a partir de inventarios forestales nacionales: Buenas prácticas para la recolección, manejo y análisis de datos" 1ra ed, CATIE: Turrialba, 2017. 